\title{
Avaliação de eventos adversos relacionados a medicamentos como indicador de implantação de um centro de informações sobre medicamentos
}

\author{
Evaluation of adverse events related to medicines as indicator of implementation of a medicinal \\ information center
}

Evaluación de eventos adversos relacionados con medicamentos como indicador de implantación de un centro de información sobre medicamentos

Benedita Leida Martins Rodrigues ${ }^{1 *}$, Vera Lucia de Azevedo Lima ${ }^{1,2}$, Jocileide de Sousa Gomes $^{3,4}$, Lizomar de Jesus Maués Moia ${ }^{1,5}$, Ingrid Magali de Souza Pimentel ${ }^{1,5}$, Cinthya Francinete Pereira Pires ${ }^{1,6}$

\section{RESUMO}

Objetivo: Analisar a frequência de eventos adversos advindos da utilização da terapia medicamentosa em um hospital referência materno-infantil do Estado do Pará. Métodos: Estudo descritivo transversal quantitativo, baseado na análise de dados retrospectivos compreendendo todas as notificações, por busca ativa ou notificação voluntária, recebida no serviço de farmacovigilância do hospital, referente aos eventos relacionados ao uso do medicamento de 24 meses compreendidos no período entre 01 de janeiro de 2015 a 31 de dezembro de 2016. Resultados: As notificações de eventos adversos, advindos da utilização da terapia medicamentosa, totalizaram 1.690 registros, dos quais 621 notificações são referentes ao serviço de farmacovigilância e 1.069 notificações do serviço de assistência farmacêutica. O setor de clínica médica obteve o maior índice, sendo 44 (51,75\%) eventos em 2015 e 335(62,49\%) em 2016. Das 85 notificações realizadas no serviço de farmacovigilância em 2015, em 22 (25,88\%) houve intervenções, em 63 (74,12\%) não ocorreu à intervenção. Em 2016 das 536 notificações 280 (52,24\%) não possuem registro de intervenção e em 256 (47,76\%) foi observado registro das intervenções. Conclusão: O estudo permitiu identificar os eventos adversos e seus fatores de risco evidenciando-se que a corresponsabilidade dos profissionais ao utilizar o sistema de medicamentos deve ser enfatizada dentro do processo de educação permanente na saúde no intuito de diminuir a incidência dos mesmos, contribuindo para a segurança dos pacientes no ambiente hospitalar.

Palavras-chave: Farmacovigilância, Medicamentos, Notificação.

\section{SUMMARY}

Objective: The study aimed to analyze the frequency of adverse events resulting from the use of drug therapy in a maternal and child hospital in the State of Pará. Method: A quantitative cross-sectional descriptive study based on the analysis of retrospective data comprising all notifications by active search or voluntary notification received at the hospital pharmacovigilance service for events related to the use of the 24-month drug included in the period between January 1, 2015 as of December 31, 2016s:. Results: The notifications of adverse events, resulting from the use of drug therapy totaled 1,690 records, of which 621 reports refer to the pharmacovigilance service and 1,069 notifications from the pharmaceutical care service. The medical clinic sector had the highest rate, with 44 (51.75\%) events in 2015 and 335 (6249\%) in 2016. Of

\footnotetext{
${ }^{1}$ Fundação Santa Casa de Misericórdia do Pará (FSCMP), Belém-Pará.*E-mail: leida-martins@hotmail.com

${ }^{2}$ Universidade Federal do Pará (UFPA), Belém-Pará.

${ }^{3}$ Centro Universitário do Estado do Pará (CESUPA), Belém-Pará.

${ }^{4}$ Secretaria Estadual de Saúde do Pará (SESPA), Belém-Pará.

${ }^{5}$ Universidade Estadual do Pará (UEPA), Belém-Pará.

${ }^{6}$ Escola Superior da Amazônia (ESAMAZ).
} 
the 85 notifications made at the pharmacovigilance service in 2015, in $22(25.88 \%)$, there were interventions, in $63(74.12 \%)$ did not occur to the intervention. In 2016 of the 536 notifications, 280 (52.24\%) did not have an intervention record and in $256(47.76 \%)$ a record of interventions was observed. Conclusion: The study allowed the identification of adverse events and their risk factors, showing that the co-responsibility of professionals in the drug system process should be emphasized within the process of permanent education in order to reduce their incidence contributing to the safety of patients in the hospital.

Key-words: Pharmacovigilance, Medicines, Notification.

\section{RESUMEN}

Objetivo: El estudio objetivó analizar la frecuencia de eventos adversos provenientes de la utilización de la terapia medicamentosa en un hospital referencia materno-infantil del Estado de Pará. Métodos: Estudio descriptivo transversal cuantitativo, basado en el análisis de datos retrospectivos que comprende todas las notificaciones, por búsqueda activa o notificación voluntaria, recibida en el servicio de farmacovigilancia de la FSCMP referente a los eventos relacionados al uso del medicamento de 24 meses comprendidos en el período comprendido entre el 1 de enero de 2015 a 31 de diciembre de 2016. Resultados: Las notificaciones de eventos adversos, provenientes de la utilización de la terapia medicamentosa, totalizaron 1.690 registros, de los cuales 621 notificaciones se refieren al servicio de farmacovigilancia y 1.069 notificaciones del servicio de asistencia farmacéutica. El sector de la clínica médica obtuvo el mayor índice, siendo $44(51,75 \%)$ eventos en 2015 y $335(6249 \%)$ en 2016. De las 85 notificaciones realizadas en el servicio de farmacovigilancia en 2015 , en $22(25,88 \%)$, hubo intervenciones, en $63(74,12 \%)$ no ocurrió a la intervención. En 2016 de las 536 notificaciones 280 (52,24\%) no tienen registro de intervención y en 256 $(47,76 \%)$ se observó registro de las intervenciones. Conclusión: El estudio permitió identificar los eventos adversos y sus factores de riesgo evidenciándose que la corresponsabilidad de los profesionales en el proceso del sistema de medicamentos debe ser enfatizada dentro del proceso de educación permanente con el fin de disminuir la incidencia de los menos contribuyendo a la seguridad de los pacientes en el ambiente hospitalario.

Palabras clave: Farmacovigilancia, Drogas, Notificación.

\section{INTRODUÇÃO}

A Farmacovigilância é a ciência que objetiva a detecção, avaliação e compreensão de efeitos adversos, além de todo e qualquer evento adverso relacionado a medicamento. Os eventos adversos, por sua vez são considerados nocivos à saúde do paciente e podem ser classificados como Reações Adversas a Medicamentos (RAM), erros de medicação, desvio de qualidade, interações medicamentosas e intoxicações (CAPUCHO HC, et al., 2011).

Segundo Melo CMM e Santos TA (2012), no âmbito hospitalar, o gerenciamento dos processos envolvidos na cadeia medicamentosa, representa um desafio extremamente complexo, visto que exige dos profissionais que compõem a equipe multiprofissional competências peculiares para a implementação de estratégias adequadas que possibilitem garantir a qualidade e eficiência no atendimento, minimizando as falhas e, consequentemente, os erros relacionados ao uso de medicamentos.

Os processos de utilização do medicamento envolvem profissionais da farmácia, medicina e enfermagem, cujas responsabilidades são complementares e definidas em legislações específicas. O médico é o responsável pela prescrição, o farmacêutico e a equipe de farmácia pela distribuição e dispensação, e a equipe de enfermagem é responsável pelo preparo, administração e o monitoramento das reações dos pacientes, sendo assim, todos os envolvidos tem a responsabilidade de prevenir e detectar eventos adversos relacionados ao uso do medicamento. "Neste sentido, o sistema de utilização de medicamentos em âmbito hospitalar constitui-se de um conjunto de processos inter-relacionados, cujo 
objetivo é a utilização dos medicamentos de forma segura, efetiva, adequada e eficiente" (CASSIANI SHB, et al., 2005).

O compromisso com a segurança do paciente permeia não apenas o uso correto e adequado dos medicamentos mas também a qualidade em todos os processos que estão envolvidos nesse uso como o planejamento, o sistema de dispensação dos medicamentos entre outros fatores, objetivando a satisfação dos clientes, a adequação dos serviços prestados e a homogeneidade dos resultados do processo (NICOLINE CB e VIEIRA RCP, 2011).

$\mathrm{Na}$ busca de se reformar a qualidade dos serviços prestados pela segurança do paciente, os setores devem buscar a interação de processos, a partir da avaliação e melhoria de sua rotina, através de estratégias que possam garantir o fornecimento e uso adequado dos medicamentos em tempo hábil e sem interrupções, estabelecendo sistemas de autocontrole que garantam a qualidade dos serviços. Pesquisas recentes mostram que a gestão pela qualidade, controle de processos, satisfação do cliente, acreditação e certificações são cada vez mais buscados para garantir a prestação de serviços de excelência (FERREIRA CAA, et al., 2013; BRUM LFS, et al., 2014).

Segundo Cohen MR (2007), existem dez elementos-chave característicos do sistema de utilização do medicamento que precisam ser avaliados para que tenhamos práticas seguras, uma vez que representam as causas dos erros de medicação, os quais são: informação sobre o paciente; informação relacionada ao medicamento; comunicação de informações sobre as drogas; rotulagem e embalagem; dispensação, armazenamento e padronização; aquisição, uso e monitoramento de dispositivos; fatores ambientais; educação dos profissionais; educação do paciente; gerenciamento de riscos e processos de qualidade.

Dentre os eventos adversos, os erros de medicação são ocorrências comuns e podem assumir proporções clínicas significativas impondo custos elevados ao sistema de saúde, logo o hospital estudado, por ser referência estadual no atendimento materno infantil e ter como visão de futuro ser um hospital Público de excelência, com reconhecimento nacional em gestão, assistência, ensino e pesquisa de forma segura, humanizada e sustentável (RELATÓRIO DE GESTÃO 2017, p.28) investe em mecanismos que tornam o processo de utilização do medicamento mais seguro, principalmente no que tange aos erros de medicação por serem erros considerados evitáveis. Vale ressaltar que o hospital, tem seus processos finalísticos voltados para a assistência, ensino e pesquisa buscando a promoção e a inclusão social com objetivo de garantir a qualidade do atendimento. As perspectivas definidas em seu Mapa Estratégico para o alcance de sua visão são: Ética, desenvolvimento humano, segurança, sustentabilidade, resolutividade, integralidade, humanização e valorização das pessoas.

Frente ao atual cenário, o hospital busca alinhar seu planejamento estratégico à cultura de segurança do paciente, tornando-se imprescindível a busca de uma reflexão crítica para reconhecer e entender o campo e a ocorrência dos eventos adversos relacionados ao uso dos medicamentos. Portanto, o presente estudo tem como objetivo analisar a frequência de eventos adversos advindos da utilização da terapia medicamentosa no hospital estudado, visando garantir a segurança do paciente através da estratégia de vigilância e educação permanente em saúde.

\section{MÉTODOS}

Estudo descritivo, transversal, quantitativo, retrospectivo baseado na análise de dados de todas as notificações, sejam por busca ativa ou notificação voluntária, recebidas no serviço de farmacovigilância do hospital referente aos eventos relacionados ao uso do medicamento de pacientes internados durante 24 meses compreendidos no período entre 01 de janeiro de 2015 a 31 de dezembro de 2016.

Foram excluídas todas as notificações recebidas e classificadas a outros fatores não relacionados a eventos advindos do uso do medicamento, tais como hemo vigilância, tecno vigilância, vacinas, alimentos.

O estudo foi realizado obedecendo aos preceitos das Diretrizes e Normas Regulamentadoras de Pesquisas Envolvendo Seres Humanos impostas pela Resolução CNS 466/12. Após anuência da gerência 
de Pesquisa e aprovação do Comitê de Ética em Pesquisa (CEP) do hospital através de submissão do projeto via Plataforma Brasil recebendo aprovação conforme protocolo 2.147.376.

\section{RESULTADOS}

Durante a realização do estudo foram computadas informações referentes aos medicamentos prescritos em 2015 e 2016 para pacientes internados que apresentaram eventos adversos notificados ao serviço de farmacovigilância através de busca ativa ou notificação voluntária.

Nesse período, as notificações de eventos adversos, advindos da utilização da terapia medicamentosa, totalizaram 1.690 registros, dos quais 621 notificações são referentes ao serviço de farmacovigilância e 1.069 notificações do serviço de assistência farmacêutica. Ressalta-se que foram considerados também os dados registrados no serviço de assistência farmacêutica, a fim de subsidiar a análise e discussão dos resultados.

Das 85 notificações realizadas no serviço de farmacovigilância, em 2015 houve intervenções apenas em 25,88\%. Em 2016, entretanto, o registro de intervenções subiu para 47,76\% (Figura 1).

Ressalta-se que do total de intervenções realizadas pelo farmacêutico junto a equipe assistencial, no período estudado, $48,92 \%$ recomendaram a suspensão do medicamento, $27,70 \%$ propuseram ajustes na prescrição e $23,38 \%$ recomendaram continuidade do uso do medicamento.

Figura 1 - Distribuição das notificações quanto a intervenção da farmacovigilância nos anos de 2015 e 2016.

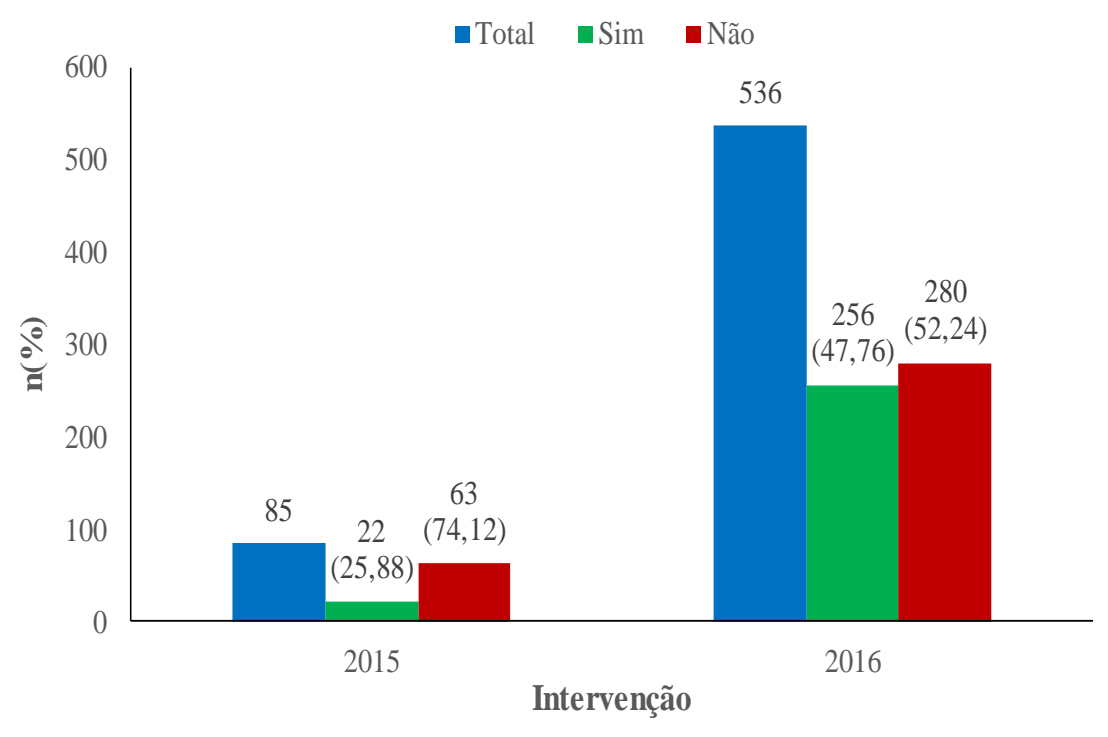

Fonte: Dados da pesquisa, 2018

Observando-se os dados da figura 2, no ano de $2015,71,76 \%$ das notificações ocorreram por busca ativa, $25,88 \%$ por notificação voluntária e em $2,35 \%$ a informação estava como ignorada. No ano subsequente, $93,47 \%$ das notificações ocorreram por busca ativa, 5,04\% foram notificações voluntárias e em 1,49\% notificações a informação estava ignorada. 
Figura 2- Distribuições dos tipos de notificações, nos anos de 2015 e 2016, do serviço de farmacovigilância.

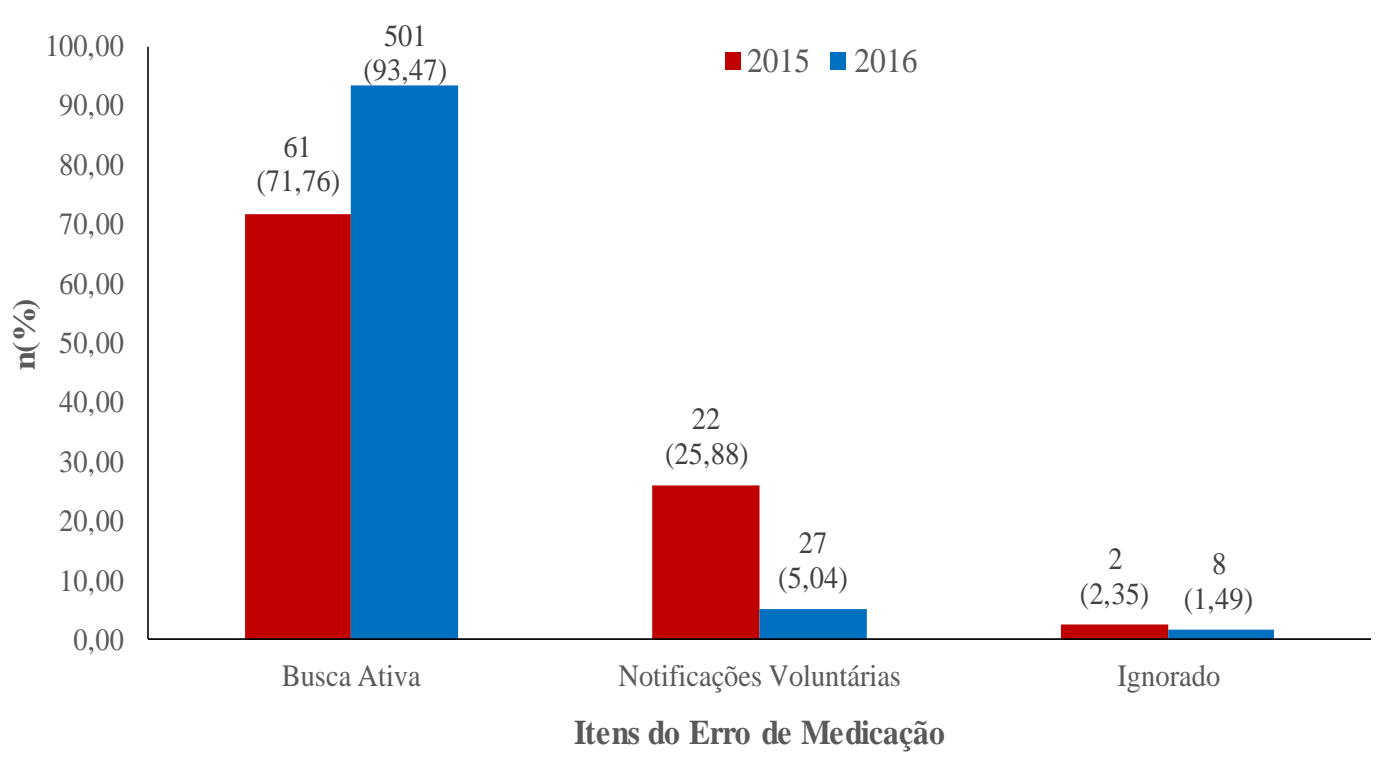

Fonte: Dados da pesquisa, 2018

No ano de 2015, do total de 148 incidentes, 109 (73,65\%) foram erro de medicação, 34 (22,97\%) casos de RAM, $3(2,03 \%)$ de inefetividade terapêutica e $2(1,35 \%)$ de queixa técnica. No ano de 2016, do total de 1.108 notificações, verificamos a frequência de 817 (73,74\%) erros de medicação, $286(25,81 \%)$ RAM e 5 $(0,45 \%)$ eventos de queixa técnica (Tabela 1$)$.

Tabela 1 - Distribuição dos incidentes em farmacovigilância, nos anos de 2015 e 2016.

\begin{tabular}{lccc}
\hline \hline \multirow{2}{*}{ Eventos Adversos } & \multicolumn{2}{c}{ Ano n (\%) } & Total \\
\cline { 2 - 4 } & $\mathbf{2 0 1 5}$ & $\mathbf{2 0 1 6}$ & \\
\hline Erros de Medicação & $109(73,65)$ & $817(73,74)$ & 926 \\
RAM & $34(22,97)$ & $286(25,81)$ & 320 \\
Inefetividade & $3(2,03)$ & - & 3 \\
Queixa Técnica & $2(1,35)$ & $5(0,45)$ & 7 \\
\hline Total & $\mathbf{1 4 8 ( 1 0 0 , 0 0 )}$ & $\mathbf{1 1 0 8 ( 1 0 0 , 0 0 )}$ & $\mathbf{1 2 5 6}$ \\
\hline \hline
\end{tabular}

Fonte: Dados da pesquisa, 2018.

Quanto aos erros de medicação, no ano de 2016, o erro de prescrição manteve-se com a maior frequência, ocorrendo em $27,0 \%$ dos casos, enquanto $23,0 \%$ foram erros de dispensação, $23,0 \%$ erros de cálculo/preparação, 26,0\% erros relacionados à rotina de administração e 1,0\% foram erros de distração (Figura 3). 
Figura 3 - Distribuições dos Erros de Medicação em farmacovigilância conforme causalidade, nos anos de 2015 e 2016.

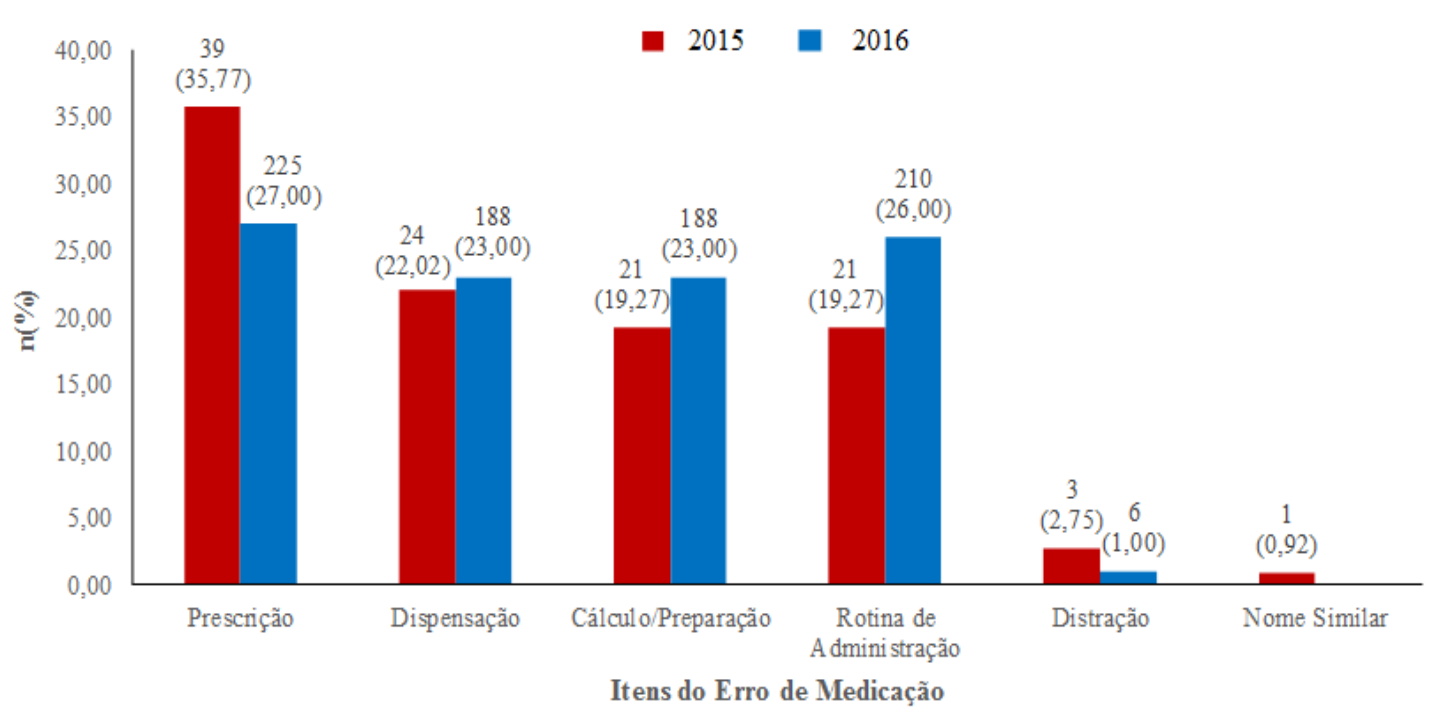

Fonte: Dados da pesquisa, 2018.

\section{DISCUSSÃO}

Ao falarem sobre erros de medicação, Rodrigues MCS, et al. (2010) e Oliveira RB, et al. (2011) afirmam que os erros relacionados a terapia medicamentosa abrangem qualquer incidente prevenível com viabilidade de causar dano ao paciente. Estando sob os cuidados dos profissionais de saúde, tais riscos podem ser associados à prática profissional, aos insumos utilizados, e/ou aos procedimentos e fluxos adotados, incluindo a construção da prescrição, rótulo, embalagem de produtos e nomenclaturas, bem como ao processo de dispensação, distribuição e monitoramento de uso dos fármacos. Os fatores de risco identificados nos processos relacionados ao uso de medicamentos foram: possibilidade de interações medicamentosas, incompatibilidades entre medicamentos e diluentes prescritos, erros de dose, risco de erros de prescrição e horários concomitantes de medicamentos incompatíveis. Dentre os fatores de riscos destacam-se aqueles relacionados aos erros de prescrição, que podem levar a outros tipos de erros como erros na dispensação e erros na administração dos medicamentos prescritos inadequadamente.

Em uma pesquisa realizada por Dalmolin GRS e Goldim JR (2013), os autores concluíram que os erros de medicação devem ser tratados em todas as suas repercussões pessoais, profissionais e institucionais. Além disso, deve-se avaliar os riscos e a possibilidade de sua ocorrência, em todo o ciclo da cadeia medicamentosa, bem como na implementação de políticas institucionais que estimulem a cultura de notificação de erros de medicação, culminando com a capacitação das equipes envolvidas. Sendo assim, há necessidade de intervenções, a fim de garantir que os erros sejam devidamente tratados e previnidos.

Para a Organização Mundial de Saúde (OMS), a subnotificação não é somente uma questão técnica, mas também psicológica, que pode ser minimizada ao se adotar procedimentos simples associado a práticas motivacionais. A meta proposta pela OMS é de 250 notificações/milhão de habitantes, porém a própria OMS afirma que até em centros de farmacovigilância bem estabelecidos, a porcentagem de reações graves notificadas não ultrapassa $10 \%$ do total de reações, sendo a subnotificação a grande limitação, logo é difícil dimensionar de forma fidedigna o problema (OMS, 2005).

Quanto à distribuição dos tipos de notificações, pôde-se observar que a busca ativa foi mais presente nos dois anos, representando $90,20 \%$ das notificações, enquanto que as notificações voluntárias 
representaram somente $7,89 \%$, demonstrando assim que a cultura de notificação dentro da instituição precisa ser fortalecida para que assim, a equipe de profissionais da saúde possa ter clareza dos procedimentos de notificação e entendam que ao notificar estarão contribuindo efetivamente para que medidas educativas possam ser implementadas no intuito de tornar suas práticas mais seguras ao utilizar o medicamento.

Vaz IR, et al. (2010) em sua pesquisa, concluiu que a realização de ações educativas aumenta a quantidade e a relevância das notificações voluntárias de reações adversas a medicamentos por parte dos farmacêuticos do norte de Portugal, por um período de até quatro meses. Diante desses resultados, foi proposto pelos autores da pesquisa que a sensibilização seja realizada a cada quatro meses, uma vez que, após esse período observa-se perda de importância das ações.

Corroborando com este pensamento, a pesquisa de Richards DTL e Graham P (2003) demonstra que os profissionais potencialmente notificadores precisam de formações regulares para que possam manter-se no processo de Farmacovigilância. A notificação voluntária é considerada cientificamente com pouca expressividade em relação a outros métodos, como: monitorização de prescrição evento, estudos de coortes ou de caso controle e ensaios clínicos controlados aleatórios. Todavia, os autores consideram as notificações voluntárias de RAM eficazes para gerar segurança, já que a notificação representa uma fonte de informação do risco dos medicamentos (VAZ IR, et al., 2010).

A pesquisa de Rodrigues MCS, et al.(2010) e Oliveira RB, et al.(2011), destaca o papel do enfermeiro no processo de prevenção de erros de medicação relacionados a rotina de aprazamento de medicamentos prescritos, evidenciando que o profissional deve conhecer as características da terapia medicamentosa, bem como as condição clínica do paciente possibilitando a prevenção de interações fármaco-fármaco e entre fármaco-alimento, incompatibilidades medicamentosas entre outros, minimizando, assim, acontecimentos relacionados a eventos adversos com medicamentos ministrados aos pacientes.

Da mesma forma, Oliveira RB, et al. (2011) e Melo CMM, Santos TA (2011) afirmam que quando um erro ocorre, o ponto mais importante não é quem cometeu o erro, mas como e porque o sistema de defesa falhou. $\mathrm{Na}$ área da saúde, principalmente para a enfermagem, a análise do processo de causa- ação tem papel de destaque, visto que os profissionais são submetidos a ações, na maioria das vezes punitivas, quando um erro de medicação é detectado. A consequência imediata é a dificuldade de identificação do erro, pois o erro ocorrido é velado por todos que nele estão envolvidos. Esta atitude perante o erro parte de uma visão individual e considera a falha humana como a única causa para ocorrência do erro.

Diante disto, deve-se compreender que para garantir um sistema de saúde mais seguro, torna-se fundamental a análise dos incidentes e eventos adversos advindos da terapia medicamentosa, pois estes são ótimos indicadores de qualidade que fornecem informações imprescindíveis para o planejamento em saúde (VLAYEN IR, et al., 2012).

Este estudo permitiu identificar os eventos adversos e seus fatores de risco, evidenciando-se que a corresponsabilidade dos profissionais no processo de sistema de medicamentos deve ser enfatizada dentro do processo de educação permanente em saúde no intuito de diminuir a incidência destes, contribuindo com isso para a segurança dos pacientes no ambiente hospitalar.

A qualificação dos profissionais farmacêuticos que atuam nas farmácias satélites é um fator primordial a fim de garantir que a análise das prescrições e demais ações voltadas à segurança durante a terapia medicamentosa sejam realizadas com bases técnicas. Ressalta-se a necessidade de ações de educação voltadas para os auxiliares de farmácia, com foco na segurança do uso de medicamentos, envolvendo todas as etapas do processo.

Observou-se com este estudo, que a maior frequência de erros de medicação ocorre nas etapas de prescrição e administração, ressaltando que os erros de administração podem estar subestimados pela própria limitação dos dados analisados, pois em uma elevada taxa de 39\% de notificações não foi possível saber se o erro afetou o paciente, estando a informação ignorada, lembrando que a relevância de tais 
informações deve-se ao fato de que a administração do medicamento é realizada por profissionais de enfermagem sendo a última barreira contra os erros de medicação. Já os erros de prescrição passam por outros processos de análise antes de chegar ao paciente, com mais chances de serem evitados.

\section{CONCLUSÃO}

Mediante o cenário evidenciado o estudo propôs a implantação do Centro de Informação sobre Medicamentos, com enfoque na proposta de educação permanente em saúde, para garantir a melhoria da segurança do paciente dentro do contexto hospitalar. Além disso, aperfeiçoar o fluxo das intervenções multiprofissionais frente às prescrições, dispensação, preparação e administração de medicamentos com o objetivo de reduzir os eventos adversos relacionados a medicamentos.

\section{REFERÊNCIAS}

1. AGUIAR PM, BALISA RBJ, LYRA JDP. Avaliação de indicadores de estrutura e processo de um serviço de Atenção Farmacêutica na Farmácia Popular do Brasil: um estudo piloto; Evaluation of structure and process indicators at a pharmaceutical care service of a drugstore chain in Brazil: A pilot study. Rev. ciênc. farm. básica apl, 2013; 34(3).

2. BRUM LFS, et al. Análise do cumprimento de dispositivos legais em prescrições de medicamentos sob controle especial aviadas em uma farmácia comunitária de Porto Alegre-RS. Inova Saúde, 2014; 2(2).

3. CASSIANI SHB, et al. O sistema de medicação nos hospitais e sua avaliação por um grupo de profissionais. Rev. Esc. enferm. USP. 2005; 39(3): 280-87.

4. CAPUCHO HC, ARNAS ER, CASSIANI SHBD et al. Farmacovigilância: gerenciamento de riscos de terapia medicamentosa para a segurança do paciente. São Caetano do Sul: Yendis Editora, $2011 ; 448 p$.

5. COHEN MR. Medication errors. 2 ed. Washington: American Pharmaceutical Association, Apha publications. 2006;680p.

6. DALMOLIN GRS; GOLDIM JR. Erros de Medicação em Hospitais: Considerações a Partir da Bioética. Rev. Assoc. Med. Bras., 2013; 59(2).

7. FERREIRA CAA et al. Monitoramento da gestão farmacêutica com o uso de indicadores em um hospital público. Rev. Bras. Farm. Hosp. Serv. Saúde São Paulo v, v. 4, n. 2, p. 14-18, 2013.

8. MELO CMM, SANTOS TA. Gestão participativa em saúde e enfermagem: foco no processo de trabalho. In: VALE EG, PERUZZO AS, FELLI EA. (org). Programa de Atualização em Gestão De Enfermagem/PROENF/GESTÃO.4ed. Porto Alegre: ARTMED PANAMERICANA, 2012, v.1, p 65-88.

9. NICOLINE CB, VIEIRA RCP. A Assistência farmacêutica no Sistema Único de Saúde (SUS): percepções de graduandos em Farmácia. Interface-Comunicação, Saúde, Educação, 2011; 15(39): 1127-1144.

10. OLIVEIRA RB, MELO ECP. O sistema de medicação em um hospital especializado no município do Rio de Janeiro. Esc. Anna Nery, 2011; 15(3): 480-489.

11. ORGANIZAÇÃO MUNDIAL DA SAÚDE. Monitorização da segurança do Medicamento: Diretrizes para a criação e funcionamento de um centro de farmacovigilância. Brasília: OPAS; 2005. Disponível em: http://bvsms.saude.gov.br/bvs/publicacoes/seguranca.pdf. Acesso em: 28 mar. 2018.

12. RICHARDS DTL, GRAHAM P. Do clinical practice education groups result in sustained change in GP prescribing? Fam Pract. 2003; 20(2): 199-206.

13. RODRIGUES MCS, CASTRO OL. Erros na administração de antibióticos em unidade de terapia intensiva de hospital de ensino. Revista Eletrônica de Enfermagem, 2010; 12(3): 511-9.

14. SILVA JÚNIOR E, NUNES LMN. Avaliação da Assistência Farmacêutica na atenção primária no município de Petrolina (PE); Pharmaceutical Service evaluation in primary health care from the city of Petrolina, Pernambuco, Brazil. Arq. bras. ciênc. saúde, 2012; 37(2).

15. VLAYEN A et al. Design of a medical record review study on the incidence and preventability of adverse events requiring a higher level of care in Belgian hospitals. BMC Res Notes. [S.I.], 2012; 5(468): 9.

16. VAZ IR; HERDEIRO MT, POLÓNIA J, FIGUEIRAS A. Estratégias para aumentar a sensibilidade da farmacovigilância em Portugal. Rev. Saúde Pública, 2010; 45(1). Disponível em: http://www.scielo.br/scielo.php?pid=S0034-89102011000100015\&script=sci_abstract\&tlng=es.Acesso: 24 de fev 2018. 